\title{
Using Box-Scores to Determine a Position's Contribution to Winning Basketball Games
}

Gilbert W. Fellingham

gwf@byu.edu

C. Shane Reese

reese@stat.byu.edu

Garritt L. Page

Follow this and additional works at: https://scholarsarchive.byu.edu/facpub

Part of the Statistics and Probability Commons

\section{Original Publication Citation}

Page, Garritt L. Fellingham, Gilbert W. and Reese, C. Shane (27) "Using Box-Scores to Determine a Position's Contribution to Winning Basketball Games," Journal of Quantitative Analysis in Sports: Vol. 3 : Iss. 4, Article 1.

\section{BYU ScholarsArchive Citation}

Fellingham, Gilbert W.; Reese, C. Shane; and Page, Garritt L., "Using Box-Scores to Determine a Position's Contribution to Winning Basketball Games" (2007). Faculty Publications. 245.

https://scholarsarchive.byu.edu/facpub/245 


\title{
Journal of Quantitative Analysis in Sports
}

Volume 3, Issue 4 2007 Article 1

\section{Using Box-Scores to Determine a Position's Contribution to Winning Basketball Games}

\author{
Garritt L. Page* Gilbert W. Fellingham ${ }^{\dagger}$ \\ C. Shane Reese ${ }^{\ddagger}$
}

*Iowa State University, gpage2990@yahoo.com

†Brigham Young University, gwf@ byu.edu

†Brigham Young University, reese@ stat.byu.edu

Copyright (c) 2007 The Berkeley Electronic Press. All rights reserved. 


\title{
Using Box-Scores to Determine a Position's Contribution to Winning Basketball Games*
}

\author{
Garritt L. Page, Gilbert W. Fellingham, and C. Shane Reese
}

\begin{abstract}
While it is generally recognized that the relative importance of different skills is not constant across different positions on a basketball team, quantification of the differences has not been well studied. 1163 box scores from games in the National Basketball Association during the 1996-97 season were used to study the relationship of skill performance by position and game outcome as measured by point differentials. A hierarchical Bayesian model was fit with individual players viewed as a draw from a population of players playing a particular position: point guard, shooting guard, small forward, power forward, center, and bench. Posterior distributions for parameters describing position characteristics were examined to discover the relative importance of various skills as quantified in box scores across the positions. Results were consistent with expectations, although defensive rebounds from both point and shooting guards were found to be quite important.
\end{abstract}

KEYWORDS: Bayesian hierarchical model, multiple regression

*We are grateful for the insight of two referees' whose comments markedly improved the manuscript. 
Page et al.: Skill Importance by Basketball Position

\section{Introduction}

Basketball is a sport that is becoming increasingly popular worldwide. The National Basketball Association is generally considered to be the pinnacle of competitive basketball. Bray and Brawley (2002) suggest that in order for a team to be successful on any level, team members need to recognize their roles (i.e., what particular skills they will need to exhibit) and combine them in order to play as a single unit. At the professional level, each of the five positions requires unique skills. The purpose of this paper is to help determine which skills a particular position needs to optimally contribute to a team's success in the NBA.

It seems reasonable that some skills would have varying importance according to position. (Note: In this paper, "importance" is the marginal increase in team net points per possession.) For example, a turnover from a guard could possibly be more detrimental to the outcome of the game than a turnover from the center position. A turnover from a guard can occur on the perimeter, which leads to a fast break opportunity for the opposing team. A turnover from a center generally occurs near the basket where the offense can make the transition to defense faster. Also, it seems plausible that offensive rebounds from the center position are more important to the outcome of the game than offensive rebounds from the shooting guard. A center capturing an offensive rebound usually leads to an easy basket attempt, whereas a guard gathering an offensive rebound usually results in resetting the offense, which may or may not result in another attempt at a basket. Also, each coach has a different coaching philosophy that can affect the contribution of a given position. For example, Larry Brown, former coach of the New York Knicks, is vocal about his dislike of point guards that try to score more often than pass. Under Coach Brown's system, a high scoring point guard is not desirable, and would not be likely to receive much playing time. The relative abilities of the other players on the team also need to be considered. For example, a team with a dominant center may not need as much point production from other positions.

Trninic and Dizdar (2000) developed nineteen performance characteristics and assessed their relative importance for each position. Ten professional basketball experts ranked the importance of each characteristic by position. The experts had a high degree of interobserver agreement. The criteria for guards included characteristics such as "level of defensive pressure" and "transition defense efficiency" rated highly, while power forwards and centers had "defensive and offensive rebounding efficiency" and "inside shots" rated highly. Berri (1999) linked individual NBA player statistics to team wins via an econometric model. He was primarily interested in measuring each player's "production 
of wins, or the player's marginal product". Dennis Rodman was found to be the highest win-producer for the 1997-1998 NBA regular season. Bishop and Gajewski (2004) used box-score categories along with physical characteristics to predict the potential of a collegiate basketball player to be drafted into the NBA. Using multivariate methods and logistic regression, they produced a score for each collegiate player to indicate his likelihood of being drafted. Using a cutoff score of .20, they correctly categorized undrafted players at $90 \%$ and drafted players at $78 \%$.

In this paper, we use a hierarchical Bayesian approach to model the difference in points scored as a function of the difference of ten performance categories found in box-scores of NBA games. Individual players are assumed to be a random draw from a population of individuals playing a given position. Decisions regarding the relative importance of performance category by position are made using the posterior distributions of the position parameters.

\section{Data}

The results of NBA games are summarized in a box-score (see Table 1). Through USA Today's website, http://www.usatoday.com/, we were able to obtain box-scores for the 1996-1997 NBA season. USA Today's box-scores provide the final score of the game, where the game was played, and each participating player's totals for 13 performance categories. These categories are assists (ast), steals $(\mathrm{stl})$, turnovers $(\mathrm{to})$, free throws made $(\mathrm{ftm})$, free throw percentage $(f t p)$, field goals made $(f g m)$, field goal percentage $(f g p)$, offensive rebounds (orb), defensive rebounds $(d r b)$, minutes played $(\mathrm{min})$, personal fouls $(p f)$, total points $(p t s)$, and total rebounds $(t r b)$.

Box-scores identify a player's position only if the player starts. However, players that don't start often have an impact on the outcome of the game. To include all players that participated we grouped those players that didn't start into a "bench" position. In addition, these box-scores make no distinction between a point guard and a shooting guard or between a small forward and a power forward. Since point guards and shooting guards usually have vastly different roles within the framework of the team we wanted to be able to distinguish between the two. Therefore, based on personal recollection, and using the internet as a further resource, we separated the guards into point guards and shooting guards, and the forwards into small forwards and power forwards.

In the 1996-1997 NBA season, 29 teams competed so $T=29$, and the number of opponents was $O=29$. In this study the number of players that 
Page et al.: Skill Importance by Basketball Position

Table 1: Typical USA Today NBA Box-Score

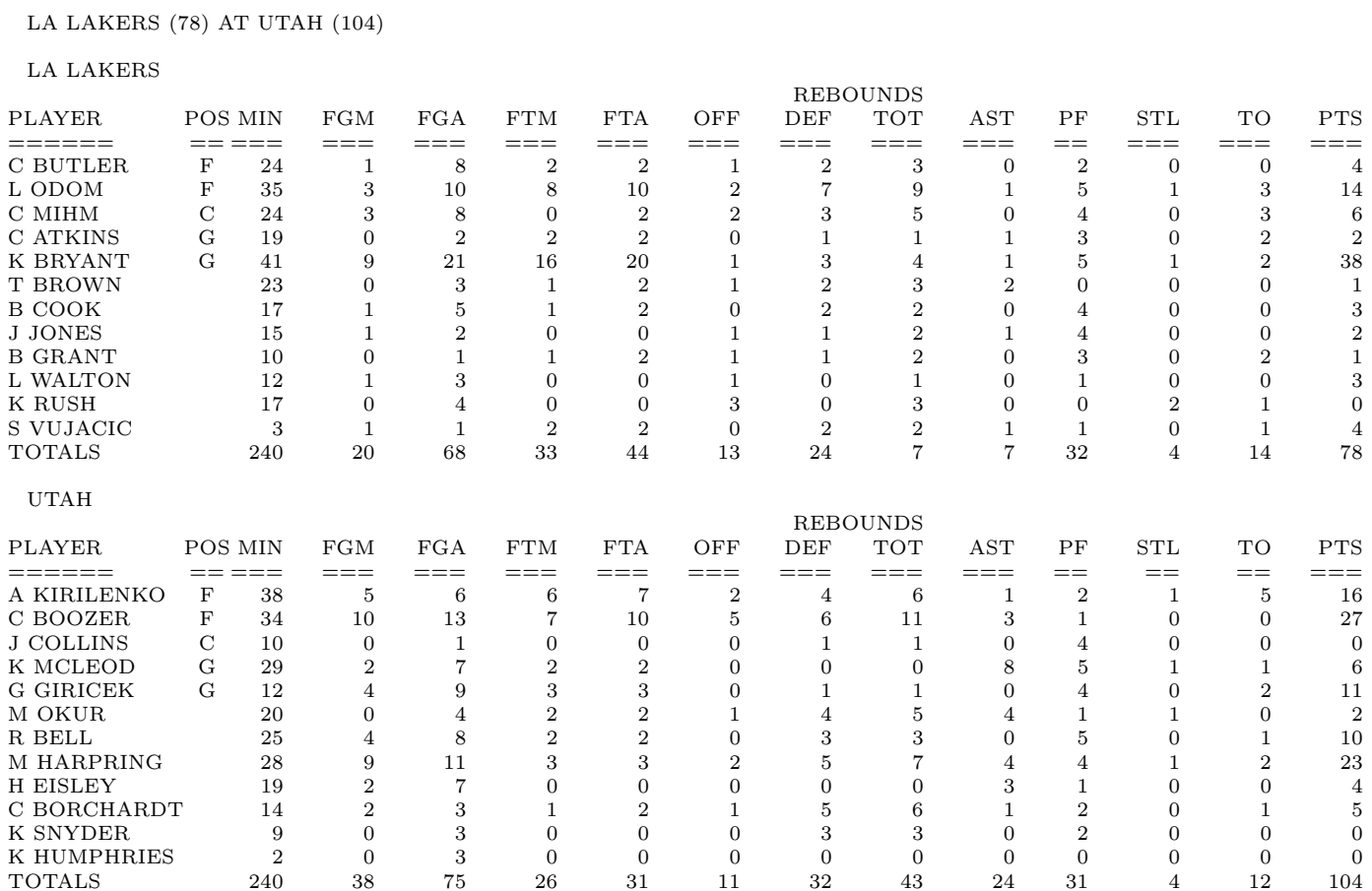

started a game was 343. Treating each team's bench as a 'player', the total number of distinct players was $P l=371$. Each of the five starters was assigned one of five positions at the beginning of a game, and with the bench position the total number of positions was $P o=6$. We assume that a player's positional assignment remained constant throughout the game. While the assumption of constant positional assignment is not necessarily an appropriate representation of what actually occurs during the course of an NBA game, analysis based on box-score data requires this assumption. The positions are: point guard (pg), shooting guard (sg), small forward (sf), power forward (pf), center (c), and bench (b). There are box-scores for 1163 games from the 1996-1997 season, so the number of games was $G=1163$.

All categories except rebounds and shooting percentages were standardized by possesions. Number of possessions is not recorded in the box-score, so we estimated the number of possessions from the box-score by

$$
p p=\frac{p m}{48} \times t p,
$$


where $p p$ is the number of possessions in which a player participated, $p m$ is the number of minutes played by the player, $t p$ is the team's possession total, and 48 is the number of minutes in an NBA game. For the bench position we used 240 in the denominator, the total number of minutes available to the bench position. To estimate $t p$ we used the following equation which incoporates categories found in the box-score:

$$
t p=f g a-o r b+t o v+0.4 f t a .
$$

Equations (1) and (2) are found in Oliver (2004).

To standardize rebounds we use individual offensive and defensive rebounding percentages, calculated as

$$
\begin{aligned}
\text { PlayerOR } \%=\frac{\text { PlayerOR }}{\% \text { Min } \times(\text { TeamOR }+ \text { OppDR })}, \\
\text { PlayerDR\% }=\frac{\text { PlayerDR }}{\% \text { Min } \times(\text { TeamDR }+ \text { OppOR })},
\end{aligned}
$$

where Player $O R$ is the total number of offensive rebounds for a player, TeamOR and TeamDR are the team's total number of offensive and defensive rebounds, $O p p O R$ and $O p p D R$ are the opponent's total number of offensive and defensive rebounds and \%MIN is the percentage of total minutes that a player participated in a game. After standardizing the box-score categories each player was paired by position (i.e. point guards from competing teams are paired and shooting guards from competing teams are paired, etc.) for each game and the differences between the matched players were computed for the standardized box-score categories (home team player minus visiting team player). These differences are the explanatory variables. We use differences to account for the differential talent levels of the opposition. For the response variable we used the difference in the final score of the game (which will be referred to as point spread).

\section{Model}

We used nine of the available box-score categories as explanatory variables in the model. Also, we used the equation $3 \mathrm{pm}=p t s-2 \mathrm{fgm}-\mathrm{ftm}$ to obtain the number of three-pointers made for a tenth variable. Unfortunately, with the present data it is impossible to determine how many three-pointers a player attempted, so three-point shooting percentage was not included in the model. Total rebounds and total points scored were also not included because they 
are linear combinations of offensive rebounds and defensive rebounds and field goals made and free throws made.

A standard multiple regression model that would determine the linear relationship between the point spread and the ten categories is

$y=\beta_{0}+\beta_{1} a s t+\beta_{2} s t l+\beta_{3} t o v+\beta_{4} 3 p m+\beta_{5} f t m+\beta_{6} f t p+\beta_{7} f g m+\beta_{8} f g p+\beta_{9} o r b+\beta_{10} d r b+\epsilon$,

where $y$ is the point spread, $\beta_{0}$ is the overall intercept, $\beta_{1}$ is the effect of the difference in assists (ast) on the point spread holding all else constant (and analogously for $\left.\beta_{2}, \ldots, \beta_{10}\right)$, and $\epsilon \sim N\left(\mathbf{0}, \sigma^{\mathbf{2}} \mathrm{I}\right)$. An implicit assumption of our model is (5) that the effects are additive. We examine the assumptions of this model in some detail in the following paragraphs. However, the additivity assumption seems to be reasonable based on preliminary studies.

Clearly, an inadequacy in equation (5) is the assumption that each $\beta_{h}$, $h=1, \ldots, 10$, is the same regardless of player or position. In addition, the way in which the data are used creates dependence in the response variable for individuals on the same team, the opponent they play, and the game in which they play; thus, the assumption of independent responses is violated. We obtain a separate $\beta$ estimate for each player and deal with the dependency issues by incorporating a Bayesian hierarchical model on the regression coefficients and adding an effect for the player's team, the opponent's team, and the game in which they are competing. Furthermore, we include an effect in the model that corresponds to where the game is being played, thus incorporating home court advantage. The full model then becomes

$$
E\left(y_{i k l m q}\right)=\gamma_{m}+\tau_{k}+\phi_{l}+\nu_{q}+\sum_{h=1}^{10} \beta_{h i} x_{h}+\epsilon,
$$

with $i=1, \ldots, 371 ; k=1, \ldots, 29 ; l=1, \ldots, 29 ; q=1, \ldots, 29 ; m=1, \ldots, 1163$; $x_{h}$ is the $h^{\text {th }}$ explanatory variable; and $\beta_{h i}$ is the $h^{\text {th }}$ regression coefficient for the $i^{\text {th }}$ player.

Each of the three parameters $\gamma, \phi$, and $\tau$ deal with a different aspect of the result. The team effect is modeled by $\tau_{k}$ so that players coming from the same team have the same intercept. This parameter can also help account for different coaching philosophies and talent level of teams. Both coaching and talent level would affect a position's role in the team framework. The opponent effect is addressed with $\phi_{l}$, the game effect is addressed by $\gamma_{m}$, and the home court advantage is addressed with $\nu_{q}$.

The point spread is symmetric around zero because each game contributes both a positive and negative response of the same magnitude, one to the 
winning team and one to the losing team. NBA games are fairly competitive, which means most games would have scoring differences close to zero and large scoring differences would not be as common. For these reasons, a Gaussian distribution is a good choice for the likelihood of the Bayesian model. Thus, we assume

$$
y_{i k l m q} \sim N\left(\theta_{i k l m q}, \sigma^{2}\right),
$$

where $\theta_{i k l m q}$ takes the form in (6).

In this study the difference in points can never be zero since a game never ends in a tie. Thus, we recognize that the normal likelihood cannot be exactly true. Nevertheless, we believe it is a reasonable approximation in this setting. For more justification of this choice of likelihood see Oliver (2004).

\section{Analysis Strategies}

In this section we discuss the reasoning behind the assignment of the prior distributions for the parameters, computational methods, and convergence diagnostics.

The values of $\beta_{h i}$ 's, $\gamma_{m}$ 's, $\phi_{l}$ 's, $\tau_{k}$ 's, and $\nu_{q}$ 's can theoretically be either positive or negative, which leads to choosing a distribution that is defined for all real numbers. A priori, little justification exists for these parameters taking on large values opposed to small ones or vice versa making symmetry an attractive choice. Thus, we select normal prior distributions for these parameters resulting in

$$
\begin{gathered}
\beta_{h i} \sim N\left(\mu_{\beta_{h, j},}, \sigma_{\beta_{h}}^{2}\right), \gamma_{m} \sim N\left(m_{\gamma}, \sigma_{\gamma}^{2}\right), \phi_{l} \sim N\left(m_{\phi}, \sigma_{\phi}^{2}\right), \tau_{k} \sim \\
N\left(m_{\tau}, \sigma_{\tau}^{2}\right), \text { and } \nu_{q} \sim N\left(m_{\nu}, \sigma_{\nu}^{2}\right) .
\end{gathered}
$$

Notice that the mean of the distribution for $\beta_{h i}$ changes according to the $j^{\text {th }}$ position of the $i^{\text {th }}$ player. Therefore, $\mu_{\beta_{1,1}}$ represents the effect of point guard assists, $\mu_{\beta_{1,2}}$ is the effect of shooting guard assists, etc. In this way the $\beta_{h i}$ 's for each player come from a "position" distribution. Thus, $\mu_{\beta_{h, j}}$ is the mean of the position distribution for position $j$ and regression coefficient $h$. These means will be the estimated position effect and the focus of this study. By letting each $\beta$ be drawn from a position distribution we are able to 'borrow strength' from all players of the same position and estimate an overall position effect. We assume $\sigma_{\beta_{h}}^{2}$ remains constant over all positions.

We use similar arguments to those above to assign hyperprior distributions. We use a Gaussian distribution for the prior distribution of $\mu_{\beta_{h, j}}$, that is,

$$
\mu_{\beta_{h, j}} \sim N\left(m_{\mu_{\beta_{h}}}, s_{\mu_{\beta_{h}}}^{2}\right) .
$$

DOI: $10.2202 / 1559-0410.1033$ 
That is, we assume that the means of the $\beta$ 's for the $j=6$ different positions are drawn from the same distribution.

The error variance, $\sigma^{2}$, is, by definition, greater than or equal to zero, necessitating a prior distribution has positive support. The inverse gamma distribution preserves the parameter space, is very flexible in its shape, and yields closed-form complete conditional distributions, making the MCMC algorithm more tractable. Therefore, the inverse gamma is a logical choice for the prior on $\sigma^{2}$. Using the same logic leads us to assign an inverse gamma distribution to $\sigma_{\beta_{h}}^{2}$ along with $\sigma_{\gamma}^{2}, \sigma_{\tau}^{2}$, and $\sigma_{\phi}^{2}$. Thus, the complete prior specification for the variance parameters is

$\sigma_{\beta_{h}}^{2} \sim I G\left(a_{\sigma_{\beta_{h}}}, b_{\sigma_{\beta_{h}}}\right), \sigma_{\gamma}^{2} \sim I G\left(a_{\sigma_{\gamma}}, b_{\sigma_{\gamma}}\right), \sigma_{\tau}^{2} \sim I G\left(a_{\sigma_{\tau}}, b_{\sigma_{\tau}}\right), \sigma_{\phi}^{2} \sim I G\left(a_{\sigma_{\phi}}, b_{\sigma_{\phi}}\right)$, and $\sigma^{2} \sim$
$I G\left(a_{\sigma}, b_{\sigma}\right)$.

\subsection{Hyperparameter Values}

This section details the selection and reasoning behind the choices for hyperparameter values. Values need to be determined for $m_{\mu_{\beta_{h}}}, s_{\mu_{\beta_{h}}}^{2}, m_{\gamma}, m_{\tau}, m_{\phi}, a_{\sigma_{\beta_{h}}}$, $b_{\sigma_{\beta_{h}}}, a_{\sigma_{\gamma}}, b_{\sigma_{\gamma}}, a_{\sigma_{\tau}}, b_{\sigma_{\tau}}, a_{\sigma_{\phi}}, b_{\sigma_{\phi}}, a_{\sigma}$, and $b_{\sigma}$.

Determination of values for $m_{\mu_{\beta_{h}}}$ is not particularly intuitive, since each slope represents the expected change in point spread given a difference of one per possession in the $h^{\text {th }}$ category, holding all other categories constant. Because this relationship is difficult to formalize even for experts, it seems reasonable that the prior specifications for these parameters should be fairly diffuse. Because an assist and a sucessful field goal attempt result in two points, it seems reasonable to believe that these two categories have the largest spread of possible values, so we choose priors for these two categories and assign these values to the prior distributions of the remaining eight categories.

A priori, the value of $\mu_{\beta_{h, j}}$ could be either positive or negative depending on the regression coefficient and the position. Hence, it seems reasonable that $m_{\mu_{\beta_{h}}}=0$. As $s_{\mu_{\beta_{h}}}^{2}$ describes the distance from zero that the values of $\mu_{\beta_{h, j}}$ could plausibly assume. We focus on $s_{\mu_{\beta_{1}}}^{2}$, which is the spread of the means for assists. An average NBA game consists of 90-100 possessions depending on team and opponent. In light of this, $\beta_{1 i}$ could take on values as large as 100-150 if all points were scored from an assist, but this is extreme and unlikely. It is more plausible that around half of field goals made are assisted, so an upper limit around 60 might be a more reasonable estimate. We choose $s_{\mu_{\beta_{1}}}^{2}=15^{2}$, which implies that $\mu_{\beta_{1, j}}$ could plausibly be assigned values up to 60 , which, in turn, allows $\beta_{1 i}$ to take on values as large as 60 . We assigned the same value to the remaining values of $s_{\mu_{\beta_{h}}}^{2}$, which complies with the desire for prior distributions for all the performance categories to be diffuse. 
Note that $\sigma_{\beta_{h}}^{2}$ measures the variability of $\beta_{h i}$. We used moment matching to find suitable parameter values. That is, we chose means and variances that reflected our belief about how the slopes might vary, and then found the parameters from the inverse gamma distribution that corresponded to the chosen means and variances. Once again, we first consider $\sigma_{\beta_{1}}^{2}$, the spread of the assist effect. It seemed reasonable that the variability of the assist effect for an individual player would not be very large relative to the point spread. Choosing $\mathrm{E}\left(\sigma_{\beta_{1}}^{2}\right)=2^{2}$ and $\operatorname{var}\left(\sigma_{\beta_{1}}^{2}\right)=3^{2}$ allows the standard deviation of $\beta_{1 i}$ to be above 10 , which is a rather large point spread. This mean and variance produce inverse gamma parameters of $a_{\sigma_{\beta_{1}}}=\frac{34}{9}$ and $b_{\sigma_{\beta_{1}}}=\frac{9}{100}$. We assign the same values to the variances of the remaining $\beta$ parameters, namely,

$$
\sigma_{\beta_{h}}^{2} \sim I G\left(\frac{34}{9}, \frac{9}{100}\right) .
$$

We assign $m_{\gamma}=m_{\phi}=m_{\tau}=m_{\nu}=0$. This seems reasonable because the four parameters $\left(\tau_{k}, \phi_{l}, \gamma_{m}\right.$, and $\left.\nu_{q}\right)$ can take on either positive or negative values depending on the team, opponent, and game.

It seemed plausible that the effects for team, opponent, and home court $(\tau, \phi$, and $\nu$ ) would be similar in their distributional form. Therefore, the same values were given to their hyperparameters. Note that $\sigma_{\tau}^{2}, \sigma_{\phi}^{2}$, and $\sigma_{\nu}^{2}$ are parameters that represent the variability that exists from team to team, which is probably larger than the within-player variance. Large deviations from zero are unlikely. Thus, it seems reasonable to find inverse gamma parameters that correspond to $\mathrm{E}\left(\sigma_{\tau}^{2}\right)=\mathrm{E}\left(\sigma_{\phi}^{2}\right)=\mathrm{E}\left(\sigma_{\nu}^{2}\right)=3^{2}$ and $\operatorname{var}\left(\sigma_{\tau}^{2}\right)=\operatorname{var}\left(\sigma_{\phi}^{2}\right)=\operatorname{var}\left(\sigma_{\nu}^{2}\right)$ $=3^{2}$. This allows the standard deviations of $\tau, \phi$, and $\nu$ take on values as large as 15, which corresponds to a rather large point spread. The values of the inverse gamma distribution that correspond with the desired mean and variance are $a_{\sigma_{\beta_{1}}}=11$ and $b_{\sigma_{\beta_{1}}}=\frac{1}{90}$. Thus,

$$
\sigma_{\tau}^{2}, \sigma_{\phi}^{2}, \sigma_{\nu}^{2} \sim I G\left(11, \frac{1}{90}\right)
$$

The effect $\gamma$ is interpreted as the point spread for a particular game given that the competing teams recorded the same number of assists per possession, steals per possession, turnovers per possession, and so on. The variance of this effect, which is the within game variance, is probably smaller than that of the team and opponent effects. Once again we use moment matching to find values of the distribution of $\sigma_{\gamma}^{2}$. We found values such that $\mathrm{E}\left(\sigma_{\gamma}^{2}\right)=(\sqrt{2})^{2}$ and $\operatorname{var}\left(\sigma_{\gamma}^{2}\right)=3^{2}$. These values would allow the standard deviation to plausibly reach values as high as 10 . Thus we choose:

$$
\sigma_{\gamma}^{2} \sim I G\left(\frac{22}{9}, \frac{173}{500}\right)
$$

DOI: $10.2202 / 1559-0410.1033$ 
Because $\sigma^{2}$ is the variability of the error term, we thought it likely that, on average, the standard deviation would be about 6 . An inverse gamma with $E\left(\sigma^{2}\right)=6^{2}$ and $\operatorname{var}\left(\sigma^{2}\right)=(2 \sqrt{5})^{2}$ would seem reasonable. Again, using moment matching, we choose:

$$
\sigma^{2} \sim I G\left(\frac{131}{25}, \frac{3}{500}\right) .
$$

For a summary of hyperparameter values see Table 2.

Table 2: Hyperparameter Values

\begin{tabular}{|ccc|ccc|}
\hline Parameter & $\mathrm{m}$ & $s^{2}$ & Parameter & $\mathrm{a}$ & $\mathrm{b}$ \\
\hline$\mu_{\beta_{1 j}}$ & 0 & 225 & $\sigma_{\beta_{1}}^{2}$ & $34 / 9$ & $9 / 100$ \\
$\mu_{\beta_{2 j}}$ & 0 & 225 & $\sigma_{\beta_{2}}^{2}$ & $34 / 9$ & $9 / 100$ \\
$\mu_{\beta_{3 j}}$ & 0 & 225 & $\sigma_{\beta_{3}}^{2}$ & $34 / 9$ & $9 / 100$ \\
$\mu_{\beta_{4 j}}$ & 0 & 225 & $\sigma_{\beta_{4}}^{2}$ & $34 / 9$ & $9 / 100$ \\
$\mu_{\beta_{5 j}}$ & 0 & 225 & $\sigma_{\beta_{5}}^{2}$ & $34 / 9$ & $9 / 100$ \\
$\mu_{\beta_{6 j}}$ & 0 & 225 & $\sigma_{\beta_{6}}^{2}$ & $34 / 9$ & $9 / 100$ \\
$\mu_{\beta_{\beta_{j}}}$ & 0 & 225 & $\sigma_{\beta_{7}}^{2}$ & $34 / 9$ & $9 / 100$ \\
$\mu_{\beta_{8 j}}$ & 0 & 225 & $\sigma_{\beta_{8}}^{2}$ & $34 / 9$ & $9 / 100$ \\
$\mu_{\beta_{9_{j}}}$ & 0 & 225 & $\sigma_{\beta_{9}}^{2}$ & $34 / 9$ & $9 / 100$ \\
$\mu_{\beta_{10 j}}$ & 0 & 225 & $\sigma_{\beta_{10}}^{2}$ & $34 / 9$ & $9 / 100$ \\
$\mathrm{~m}_{\tau}$ & 0 & & $\sigma_{\tau}^{2}$ & 11 & $1 / 90$ \\
$\mathrm{~m}_{\phi}$ & 0 & & $\sigma_{\phi}^{2}$ & 11 & $1 / 90$ \\
$\mathrm{~m}_{\nu}$ & 0 & & $\sigma_{\nu}^{2}$ & 11 & $1 / 90$ \\
$\mathrm{~m}_{\gamma}$ & 0 & & $\sigma_{\gamma}^{2}$ & $22 / 9$ & $173 / 500$ \\
& & & $\sigma^{2}$ & $131 / 25$ & $3 / 500$ \\
\hline
\end{tabular}

\subsection{Computation}

The joint posterior distribution is highly multidimensional. In order to obtain posterior distributions we used Markov chain Monte Carlo (MCMC) simulation techniques. With our choice of likelihood and prior distributions, we have complete conditional distributions that are known and easy to sample for all parameters. Because of this, we can use the Gibbs sampling algorithm as described by Gelfand and Smith (1990) to explore the posterior space and obtain draws from the posterior distribution. The complete conditional distributions were coded in FORTRAN and were used to obtain 25,000 posterior draws following a burn of 50,000 and a thinning of 80 . That is, every $80^{\text {th }}$ 
draw was kept after the initial 50,000 draws were discarded until 25,000 draws were obtained.

\subsection{Convergence Diagnostics}

Checking the convergence of Markov chains is a difficult task in models that have a large number of parameters. Time series plots were used to assess the mixing of the chains. To check convergence, we use the criteria as explained by Raftery and Lewis (1992) and their gibbsit function that can be used in the statistical software package R. All parameters in the model met the criteria set forth by Raftery and Lewis.

\section{Results}

In this section we compare the ten box-score categories for the six positions. In the following discussion, if it is not explicitly stated that the result for an effect is in the presence of all other effects then it is implied. In addition, the following results are recommendations for playing positions beyond what one would think is their normal roll. It is obvious, for example, that a center that solely focuses on steals and disregards his rebounding duties would be counterproductive. Hence, what follows is a discussion of the marginal effect of the ten box-score categories by position. Also, recall that each effect is a difference of the positional matchup. That is, if a point guard gets one more assist per 100 possessions than his opponent, it is worth .3532 points in point spread holding all other positions and categories constant.

\subsection{Positional Performance}

Our goal was to determine which skills were most important by position and the effect these skills have on the outcome of the game. In light of this, we now focus our attention on $\mu_{\beta_{h, j}}$.

For ease of interpretation, we reparameterized the model so that the units of $\mu_{\beta_{h, j}}$ that are per-possession parameters are expressed in terms of per-100possessions. Because most comparisons in NBA basketball are done on a per100-possessions basis (see Oliver (2004)), we interpret the results as the point spread change given the difference in assists per-100-possessions, the difference in steals per-100-possessions, and so forth. Also, we reparameterized shooting percentage (free throw percentage (ftp) and field goal percentage (fgp)) and rebound percentage effects so we can interpret the effects as the average 
point spread change given $1 \%$ increase in shooting percentage or rebounding percentage, holding all other effects constant.

Figures 1 and 2 provide density plots of the $\mu_{\beta_{h, j}}$ 's. Table 3 contains a summary of the posterior distributions to these parameters. These figures and tables reveal some interesting associations. Notably, the posterior distributions for the "bench" position are less variable than starting positions because the results for all players not starting were combined to represent this position. This, of course, provided more possessions and hence more data for this position.

The following interpretations are made in the context of the NBA. Because college, high school, and international basketball are different games in many respects, applying these results to leagues other than the NBA would be problematic. However, depending on available data, methodologies are transferable.

For all five positions, out-assisting the opponent has a very positive impact. A result that was somewhat unexpected was that a small forward out-assisting his opponent on average was the most beneficial to the team. In fact, the small forward assist effect has the largest positive impact among all the position boxscore category combinations.

Something that was not foreseen was how important it was for the center position to record more steals than his opponent. A center that gets one more steal per-100-possessions than his opponent gives his team a 0.379 -point advantage on average. Although the number of steals is not a perfect defensive statistic, (players with a high number of steals tend to gamble a bit on defense), it does give an indication of the relative athleticism of a player. Having an athletic center is beneficial on defense.

Obviously, turnovers are detrimental to the effectiveness of any offense. This is clearly captured in the model since the effects of committing a turnover for all positions is both large and negative. It is interesting that turnovers for small forwards has the most negative effect and that assists for small forwards has the most positive effect. It appears that having a small forward that can pass well and protect the basketball well is highly desirable.

Free throw percentage was not a significant effect for any of the six positions, but free throws made was significant for both the guard position and the bench position.

Having a better field goal percentage than the opposition is significant for all positions, but making more field goals than the opposition is only significant for the center position. Shooting a better field goal percentage than the opposition for players that play a position that requires them to be farther from the basket has the largest effect. Both guards and small forward field 
Journal of Quantitative Analysis in Sports, Vol. 3 [2007], Iss. 4, Art. 1

Figure 1: Posterior Distributions for assists, steals, turnovers, three-pointers made, and free throws made for each position
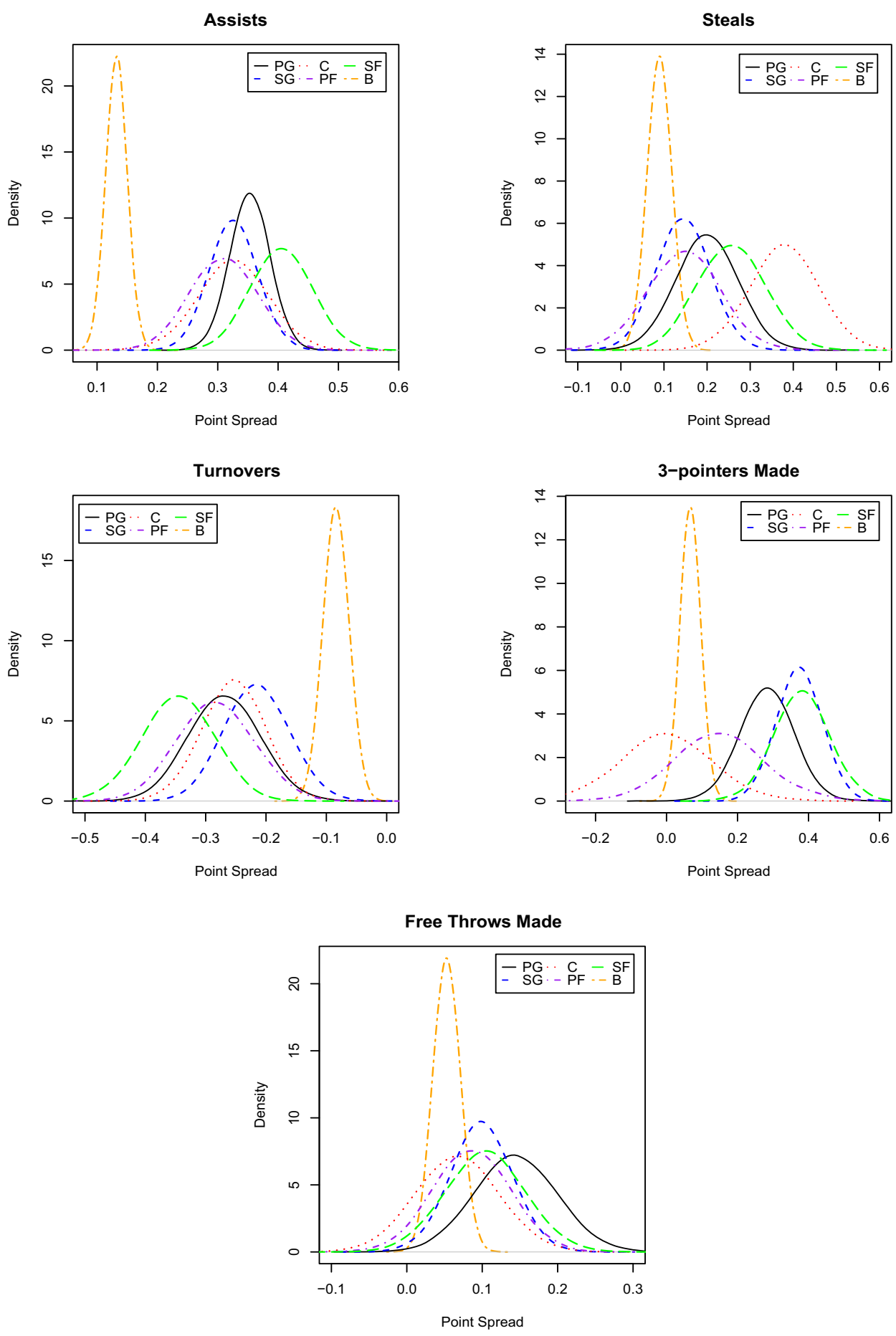

http://www.bepress.com/jqas/vol3/iss4/1 
Page et al.: Skill Importance by Basketball Position

Figure 2: Plots of posterior distributions of parameters for free throws made, free throw percentage, field goals made, and field goal percentage for each position
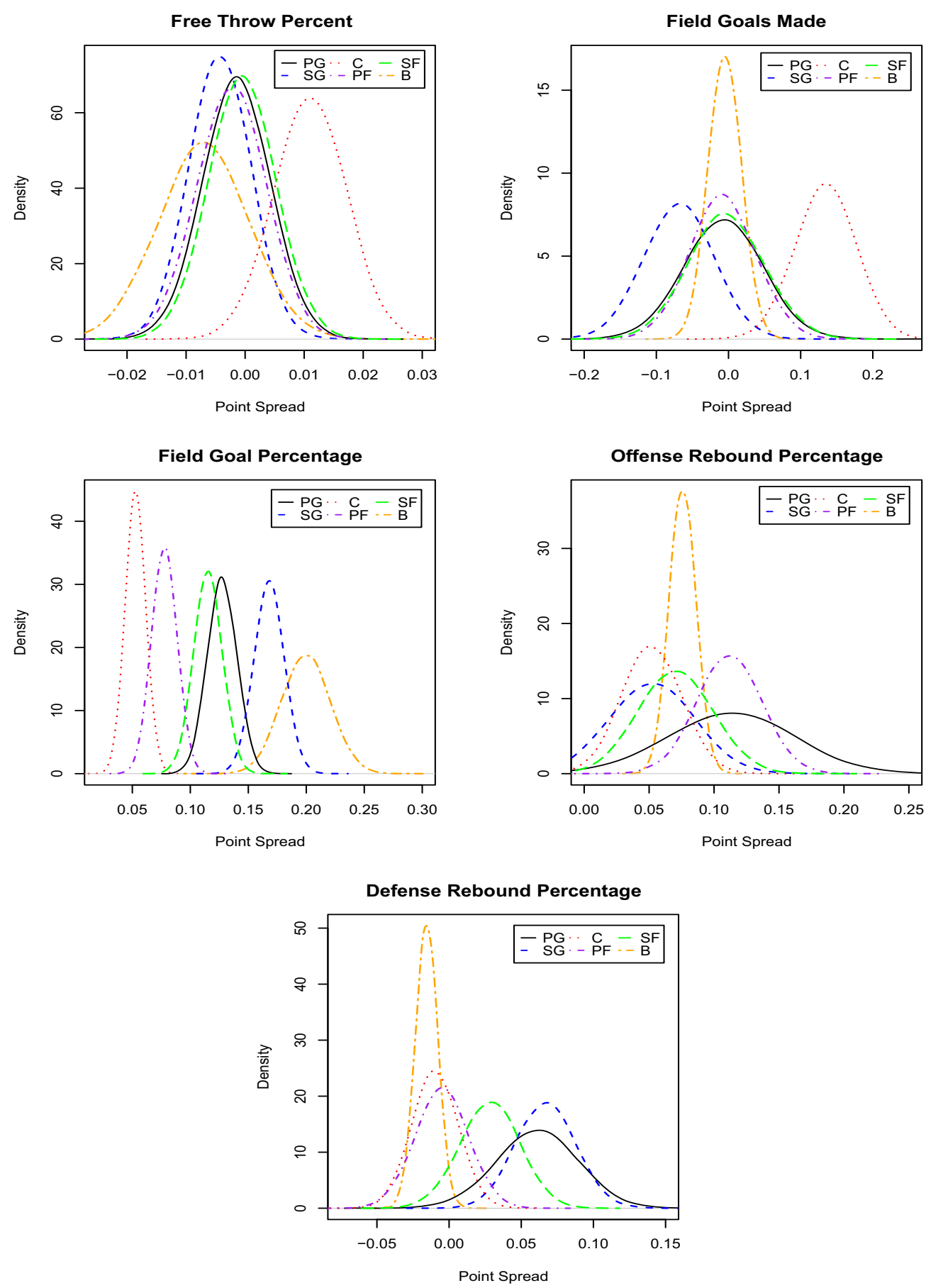

Published by The Berkeley Electronic Press, 2007 
Table 3: Posterior means, standard deviations, and 95\% HPD credible intervals of the positional categories

\begin{tabular}{|c|c|c|c|c|c|c|c|c|c|}
\hline Position & Mean & StdDev & $\begin{array}{c}2.5 \% \\
\text { LHPD } \\
\end{array}$ & $\begin{array}{r}97.5 \% \\
\text { UHPD }\end{array}$ & Position & Mean & StdDev & $\begin{array}{c}2.5 \% \\
\text { LHPD } \\
\end{array}$ & $\begin{array}{r}97.5 \% \\
\text { UHPD }\end{array}$ \\
\hline \multicolumn{5}{|c|}{$\operatorname{Assist}\left(\mu_{\beta_{1}}\right)$} & \multicolumn{5}{|c|}{$\operatorname{Steals}\left(\mu_{\beta_{2}}\right)$} \\
\hline Point Guard & 0.3532 & 0.0328 & 0.2883 & 0.4175 & Point Guard & 0.1993 & 0.0712 & 0.0559 & 0.3341 \\
\hline Shooting Guard & 0.3260 & 0.0396 & 0.2449 & 0.4008 & Shooting Guard & 0.1439 & 0.0625 & 0.0240 & 0.2671 \\
\hline Center & 0.3254 & 0.0580 & 0.2160 & 0.4463 & Center & 0.3793 & 0.0791 & 0.2189 & 0.5302 \\
\hline Power Forward & 0.3092 & 0.0552 & 0.2019 & 0.4167 & Power Forward & 0.1458 & 0.0845 & -0.0203 & 0.3104 \\
\hline Small Forward & 0.4054 & 0.0503 & 0.3060 & 0.5020 & Small Forward & 0.2570 & 0.0766 & 0.1073 & 0.4043 \\
\hline Bench & 0.1321 & 0.0176 & 0.0970 & 0.1662 & Bench & 0.0895 & 0.0280 & 0.0347 & 0.1442 \\
\hline \multicolumn{5}{|c|}{ Turn Overs $\left(\mu_{\beta_{3}}\right)$} & \multicolumn{5}{|c|}{3 -point field goals made $\left(\mu_{\beta_{4}}\right)$} \\
\hline Point Guard & -0.2687 & 0.0586 & -0.3815 & -0.1531 & Point Guard & 0.2828 & 0.0757 & 0.1341 & 0.4327 \\
\hline Shooting Guard & -0.2153 & 0.0535 & -0.3211 & -0.1125 & Shooting Guard & 0.3740 & 0.0635 & 0.2474 & 0.4963 \\
\hline Center & -0.2538 & 0.0524 & -0.3573 & -0.1521 & Center & -0.0014 & 0.1290 & -0.2602 & 0.2509 \\
\hline Power Forward & -0.2836 & 0.0628 & -0.4047 & -0.1604 & Power Forward & 0.1495 & 0.1282 & -0.1080 & 0.4021 \\
\hline Small Forward & -0.3454 & 0.0591 & -0.4615 & -0.2307 & Small Forward & 0.3810 & 0.0784 & 0.2274 & 0.5386 \\
\hline Bench & -0.0839 & 0.0212 & -0.1251 & -0.0421 & Bench & 0.0671 & 0.0287 & 0.0116 & 0.1245 \\
\hline \multicolumn{5}{|c|}{ Free Throws Made $\left(\mu_{\beta_{5}}\right)$} & \multicolumn{5}{|c|}{ Free Throw Precentage $\left(\mu_{\beta_{6}}\right)$} \\
\hline Point Guard & 0.1443 & 0.0541 & 0.0386 & 0.2500 & Point Guard & -0.0014 & 0.0056 & -0.0123 & 0.0098 \\
\hline Shooting Guard & 0.0989 & 0.0409 & 0.0204 & 0.1821 & Shooting Guard & -0.0043 & 0.0051 & -0.0139 & 0.0062 \\
\hline Center & 0.0668 & 0.0544 & -0.0405 & 0.1725 & Center & 0.0110 & 0.0060 & -0.0009 & 0.0227 \\
\hline Power Forward & 0.0857 & 0.0510 & -0.0135 & 0.1862 & Power Forward & -0.0023 & 0.0058 & -0.0136 & 0.0090 \\
\hline Small Forward & 0.1039 & 0.0525 & -0.0018 & 0.2044 & Small Forward & -0.0005 & 0.0056 & -0.0112 & 0.0108 \\
\hline Bench & 0.0528 & 0.0176 & 0.0176 & 0.0865 & Bench & -0.0070 & 0.0075 & -0.0219 & 0.0075 \\
\hline \multicolumn{5}{|c|}{ Field Goals Made $\left(\mu_{\beta_{7}}\right)$} & \multicolumn{5}{|c|}{ Field Goal Percentage $\left(\mu_{\beta_{8}}\right)$} \\
\hline Point Guard & -0.0071 & 0.0544 & -0.1143 & 0.0993 & Point Guard & 0.1275 & 0.0126 & 0.1035 & 0.1524 \\
\hline Shooting Guard & -0.0691 & 0.0479 & -0.1635 & 0.0236 & Shooting Guard & 0.1680 & 0.0129 & 0.1425 & 0.1929 \\
\hline Center & 0.1345 & 0.0416 & 0.0551 & 0.2172 & Center & 0.0527 & 0.0088 & 0.0351 & 0.0696 \\
\hline Power Forward & -0.0081 & 0.0453 & -0.0936 & 0.0846 & Power Forward & 0.0776 & 0.0108 & 0.0570 & 0.0990 \\
\hline Small Forward & -0.0033 & 0.0520 & -0.1036 & 0.0996 & Small Forward & 0.1152 & 0.0122 & 0.0913 & 0.1391 \\
\hline Bench & -0.0051 & 0.0226 & -0.0484 & 0.0399 & Bench & 0.1997 & 0.0208 & 0.1599 & 0.2411 \\
\hline \multicolumn{5}{|c|}{ Offensive Rebounds $\%\left(\mu_{\beta_{9}}\right)$} & \multicolumn{5}{|c|}{ Defensive Rebounds\% $\%\left(\mu_{\beta_{10}}\right)$} \\
\hline Point Guard & 0.1129 & 0.0482 & 0.0162 & 0.2062 & Point Guard & 0.0611 & 0.0280 & 0.0045 & 0.1140 \\
\hline Shooting Guard & 0.0522 & 0.0322 & -0.0118 & 0.1142 & Shooting Guard & 0.0670 & 0.0205 & 0.0260 & 0.1062 \\
\hline Center & 0.0519 & 0.0229 & 0.0073 & 0.0966 & Center & -0.0098 & 0.0159 & -0.0407 & 0.0212 \\
\hline Power Forward & 0.1116 & 0.0247 & 0.0638 & 0.1608 & Power Forward & -0.0051 & 0.0183 & -0.0402 & 0.0318 \\
\hline Small Forward & 0.0703 & 0.0283 & 0.0140 & 0.1246 & Small Forward & 0.0283 & 0.0205 & -0.0113 & 0.0689 \\
\hline Bench & 0.0758 & 0.0102 & 0.0560 & 0.0959 & Bench & -0.0156 & 0.0078 & -0.0309 & -0.0004 \\
\hline
\end{tabular}

goal percentages have more impact on the outcome of a game than the other positions. Thus, having a player that shoots well at these three positions is very beneficial to a team; having a bench that shoots well is also beneficial.

Offensive rebounds are important for all positions but the shooting guard. This follows conventional thought since an offensive rebound results in another 
opportunity to score. In fact it is somewhat strange that offensive rebounds for a shooting guard are not significant. What is somewhat unexpected is that defensive rebounds are only significant for the guard positions, so a center out-defensive rebounding his opponent is not as important as a point guard out-defensive rebounding his opponent, relatively speaking. This doesn't suggest that a center can disregard rebounding, only that a point guard should emphasize defensive rebounding.

Another interesting observation is that out of the three categories that guarantee scored points (assists, field goals made, and free throws made) only assists are significant for all positions. And the effect for each position out assisting their opponent is by far the greatest. That is, for each position, out assisting the opponent is more important than making more field goals than the opponent. This suggests that having a group of players play as a single unit increases the chances of winning a game.

In general the results from assists, steals, turnovers, and field goal percentages are significant for all six positions. These categories are representative of a well-rounded basketball player. Thus, having a player at each position that can perform reasonably well in all aspects of the game is desirable. This is a reflection of how the NBA game has evolved in that past few years. Players that are able to perform multiple tasks and/or play multiple positions are becoming more desirable.

\section{Conclusions}

In summary, the point spread of a basketball game increases if all five positions have more offensive rebounds, out-assist, have a better field goal percentage, and fewer turnovers than their positional opponent. These results are certainly not surprising. Some trends that were somewhat more surprising were the importance of defensive rebounding by the guard positions and offensive rebounding by the point guard. These results also show the emphasis the NBA places on an all-around small forward.

As the granularity of the data collected by basketball teams increases, results from studies of this type can help basketball coaches optimize their probability of winning basketball games by organizing practices that are customized to develop the skills which have the most impact on game outcome for each position. It also may be that these results could be used by coaches to help exploit positional matchups in specific games. Further research along these general lines, but with more detailed individual information, could be used to help coaches optimally construct their teams. 
Journal of Quantitative Analysis in Sports, Vol. 3 [2007], Iss. 4, Art. 1

\section{References}

Berri, D. (1999), "Who is "Most Valuable"? Measuring the Player's Production of Wins in the National Basketball Association," Managerial and Decision Economics, 20, 411-427.

Bishop, T. and Gajewski, B. J. (2004), "Drafting a Career in Sports: Determining Underclassmen College Players' Stock in the NBA Draft," chance, $17,9-12$.

Bray, S. R. and Brawley, L. R. (2002), "Role Efficacy, Role Clarity, and Role Performance Effectiveness," Small Group Research, 33, 233-253.

Gelfand, A. and Smith, A. F. M. (1990), "Sampling-based Approaches to Calculationg Marginal Densities," Journal of the American Statistical Association, 85, 398-409.

Oliver, D. (2004), Basketball on Paper: Rules and Tools for Performance Analysis, Dulles, VA: Brassey's Inc.

Raftery, A. E. and Lewis, S. (1992), Bayesian Statistics, Oxford, chap. How Many Iterations in the Gibbs Sampler?, pp. 765-776.

Trninic, S. and Dizdar, D. (2000), "System of the performance evaluation criteria weighted per positions in the basketball game," Collegium antropologicum., 24, 217-234. 